\title{
Shared Models and Open Infrastructures for the Smart City Internet of Things Based on the Semantic Web
}

\author{
Mouad Banane ${ }^{1}$, Abdessalam Belangour ${ }^{2}$ \\ 1,2University Hassan 2, Morocco \\ ${ }^{1}$ mouadbanane@gmail.com \\ ²belangour@gmail.com
}

\begin{abstract}
Contemporary cities face many challenges: energy, ecological, demographic or economic. To answer this, technological means are implemented in cities through the use of sensors and actuators. These cities are said to be smart. Currently, smart cities are operated by actors who share neither their sensor data nor access to their actuators. This situation is called vertical: each operator deploys its own sensors and actuators and has its own IT infrastructure hosting its applications. This leads to infrastructure redundancy and ad-hoc applications to oversee and control an area of the city. A trend is to move towards a so-called horizontal situation via the use of an open and shared mediation platform. Sensor data and access to the actuators are shared within this type of platform, allowing their sharing between the different actors. The costs of infrastructure and development are then reduced. This work is part of such a context of horizontalization, within an open and shared platform, in which we propose: 1) a layer of abstraction for control and supervision of the city, 2) a competition control mechanism handling conflict cases based on the RDF (Resource Description Framework) semantic Web standard, 3) a coordination mechanism promoting the reuse of actuators using ontology, 4) an implementation of our work by a proof of concept. The abstraction we propose is based on models from reactive systems. They aim to be generic and represent the invariant of the smart city: the physical elements. They allow applications to control and supervise the city. To facilitate the development of applications we standardize the interface of our models. Since these applications may have realtime constraints, especially those that have control objectives, we propose to take advantage of the distributed architecture of this type of platform. Given the sharing of the actuators, we have identified that conflicts can arise between applications. We propose a mechanism of competition control to deal with these cases of conflicts. We have also identified that a coordination mechanism must be offered to applications wishing to perform atomic control operations. Such a mechanism promotes the reuse of the actuators present in the city. Finally, we implemented our proposals around a proof of concept, including several use cases, to demonstrate our work.
\end{abstract}

Keywords: Smart city; Semantic web, RDF

Cite this article as: Banane M., Belangour A., "Shared Models and Open Infrastructures for the smart City Internet of Things based on the Semantic Web", International Conference on the $4^{\text {th }}$ Game Set and Match (GSM4Q-2019), Doha, Qatar, 6-7 February 2019, https://doi.org/10.29117/gsm4q.2019.0033 